Ann. Biol. anim. Bioch. Biophys., I967, 7 (2), 2I3-218.

\title{
NOTE SUR LES EFFETS DE L'INFUSION PERMANENTE D'UN MÉLANGE D'ACIDES GRAS VOLATILS DANS LE RUMEN DU VEAU APRÈS LE SEVRAGE
}

\author{
M. VERMOREL et B. GAUSSERES \\ Station d'Étude des Métabolismes, \\ Centre de Recherches zootechniques et vétérinaires sur les Ruminants. \\ 63 - Theix par Saint-Genès-Champanelle \\ Institut national de la Recherche agronomique
}

\section{INTRODUCTION}

Chez le Ruminant, 1'utilisation de 1'énergie métabolisable dépend de la composition de la ration et de la fonction physiologique qu'elle assure. Les études de BLAXTER et al. (I957 à I964) ont mis en évidence les variations de rendement énergétique des principaux produits terminaux de la digestion pour l'entretien, l'engraissement et la lactation.

Aucune recherche de ce type n'a été entreprise sur le jeune Ruminant en taison de la difficulté à appliquer ces méthodes à un animal en croissance. Avant d'aborder cette étude, nous avons dû résoudre de nombreux problèmes techniques : infusion permanente et prolongée, dans le rumen, de quantités d'acides gras volatils (A. G. V.) compatibles avec la précision des mesures de calorimétrie indirecte, prélèvements réguliers d'échantillons, analyses systématiques, comportement des animaux, etc. Cette note est relative à une partie de ces problèmes.

\section{MATÉRIEL ET MÉTHODES}

Deux veaux Frisons ( ${ }^{\circ} 43$ et 44 ) ont été sevrés progressivement au cours de leur deuxième mois ; une canule du rumen a été posée à chacun, à l'âge de 45 jours. Au début de l'infusion, les veaux étaient âgés d'environ 4 mois et pesaient respectivement 95 et $80 \mathrm{~kg}$.

Deux aliments complets $W_{1}$ et $W_{2}$ (tabl. I), présentés sous forme agglomérée ont été distribués successivement, l'aliment $W_{1}$ pendant la période préliminaire et l'aliment $W_{2}$, plus riche en azote, à partir de 8 jours avant la période d'infusion et jusqu'à la fin de l'expérience. Pour éviter les météorisations, nous avons été conduits à donner de la paille de blé. L'eau était en permanence à la disposition des animaux. L'aliment a été distribué, ad libitum, deux fois par jour $(7 \mathrm{~h} 30 \mathrm{et} \mathrm{I} 8 \mathrm{~h})$ et la paille (2oo $\mathrm{g}$ par jour) présentée de II h à I4 h. Les quantités d'aliment, de paille, d'eau, distríbuées et refusées, ont été pesées pour chaque distribution. 
Les veaux étaient installés en cage à bilan. Des échantillons ( 40 à $100 \mathrm{ml}$ ) de fluide du rumen ont été prélevés par l'intermédiaire d'une éponge placée dans la partie inférieure du rumen, sans ouvrir la canule (GAUSSERES (1965). Le $\mathrm{pH}$ était immédiatement déterminé et l'échantillon refroidi dans l'alcool. Les prélèvements ont été effectués habituellement à $8 \mathrm{~h} 30-9 \mathrm{~h}$ et $\mathrm{I} 7 \mathrm{~h} 30$.

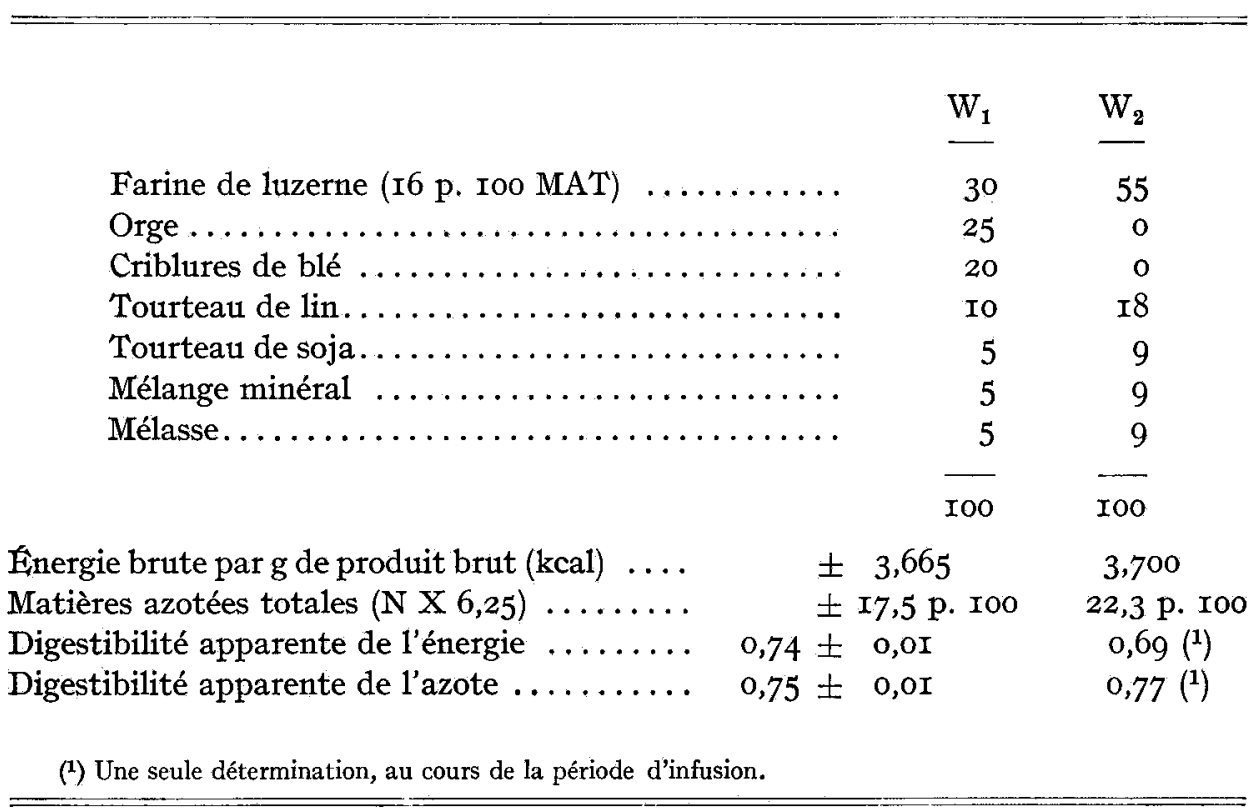

Les A. G. V. dilués dans 3 litres d'eau $(\mathrm{pH}=2,0)$ ont été infusés en permanence et à débit constant, à l'aide d'une pompe digitale. Pous éviter de modifier les fermentations du rumen, le mélange dA. G. V. était voisin de celui présent dans le rumen pendant la période préliminaire (aliment $W_{1}$ ) en pourcentage molaire : acides acétique $5 \circ$, propionique 35 , butyrique 15 .

Les quantités dA. G. V. infusées ont été augmentées, en fonction des réactions des animaux, jusqu'à $200 \mathrm{~g}$ par jour ; cette infusion a été poursuivie pendant $\mathrm{I} 7$ jours chez le veau $\mathrm{n}^{\circ} 43$ et I I jours chez le veau $\mathrm{n}^{\mathrm{O}} 44$. Une période de contrôle a été prévue en fin d'expérience avec des quantités d'aliment $\mathrm{W}_{2}$ distribuées voisines de celles consommées pendant la période d'infusion.

L'acidité du fluide du rumen a été déterminée, en double, par titration après distillation des A. G. V. (FriedemanN, I938). Les A. G. V. ont été séparés par chromatographie en phase gazeuse (JAMES et MARTIN 195I) et dosés par titration automatique après avoir été préparés suivant la méthode de ElsDen (1946) ou suivant la méthode de TiLLey (1964). L'acide butyrique et les acides gras supérieurs ont été dosés ensemble.

\section{RÉSULTATS ETT DISCUSSION}

Les principaux résultats relatifs au veau $n^{\circ} 43$ : quantités d'aliment et d'eau consommées, $\mathrm{pH}$, concentrations en $\mathrm{A}$. G. V. ont été regroupés dans la figure I. Le veau $n^{0} 43$ a bien supporté cette infusion prolongée alors que le veau $n^{0} 44$, moins vigoureux, a été assez choqué. 
En tenant compte de l'évolution générale des quantités consommées, on peut considérer que 1'infusion de $200 \mathrm{~g}$ du mélange de $\mathrm{A}$. G. V. a réduit de $35 \mathrm{p}$. Ioo les quantités d'aliment $W_{2}$ consommées par le veau $n^{\circ} 43$ et de plus de $5^{\circ} p$. Ioo (à partir de $I, 8 \mathrm{~kg}$ ) celles du veau $\mathrm{n}^{\circ} 44$. En ce qui concerne le veau $\mathrm{n}^{\circ} 43$, la ration aurait dû fournir $5650 \mathrm{kcal}$ de $E D_{c}\left(E D_{c}=E D-E_{\mathbf{c H}_{4}} \times I, 8\right)\left({ }^{1}\right)$, au cours de la période d'infusion; en fait, la ration n'a apporté que $3600 \mathrm{kcal}$ de $E D_{c}$ et les A. G. V. infusés $900 \mathrm{kcal}$, soit un déficit de I I50 kcal (20 p. roo) de $\mathrm{E}_{\mathrm{c}} \mathrm{D}_{\mathrm{c}}$ par rapport à la ration normale. Les $\mathrm{A}$. G. V. infusés ont couvert $44 \mathrm{p}$. roo de la différence de $E_{t} D_{c}$.

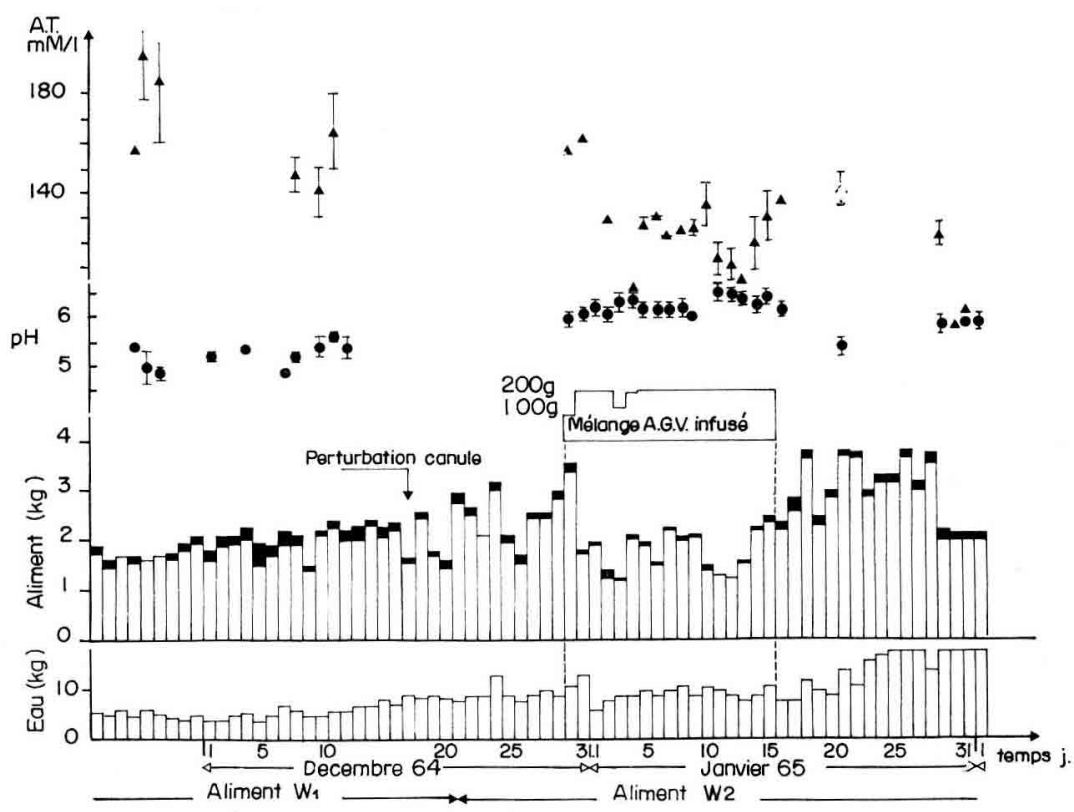

FIGURE 1. - Veau no43. Évolution des quantités d'aliment $\left(\mathrm{W}_{1}\right.$ et $\mathrm{W}_{2}$, paille) et d'eau consommées des $p H\left({ }^{\bullet}\right)$ et des acidités totales $(\mathbf{\Lambda})$ moyens journaliers avec leurs extrêmes

On peut estimer la quantité d'énergie fournie sous forme de A. G. V. soit par l'infusion, soit par l'aliment $W_{2}$, en faisant l'hypothèse que la production de $A . G . V$. représente $62 \mathrm{p}$. Ioo de l'énergie digestible (BERGMAN et al., I965). Le veau $\mathrm{n}^{\circ} 43$ a eu à sa disposition pendant la période d'infusion $3680 \mathrm{kcal}$ sous forme de $\mathrm{A}$. G. V. (2 780 provenant de 1 'aliment $\mathrm{W}_{2}$ et 900 de 1 'infusion) alors que, sans infusion, 1'aliment $\mathrm{W}_{2}$ consommé aurait fourni $4380 \mathrm{kcal}$, soit un déficit de $700 \mathrm{kcal}$ (I5 p. IOo) en A. G. V.

Avant l'infusion, la moyenne des $\mathrm{pH}$ du fluide du rumen, calculée sur les deux veaux, était faible $(5,2)$ et celle des concentrations en $A$. G. V. importante ( $160 \mathrm{mM} / 1$ ) avec l'aliment $\mathrm{W}_{1}$; les pourcentages molaires moyens étaient les suivants pour les

(1) $\mathrm{ED}_{\mathrm{c}}=$ énergie digestible corrigée d'après la formule proposée par BLAXTER (Ig62 )pour le calcul de l'énergie métabolisable corrigée. 
acides acétique, propionique et supérieurs : veau $n^{\circ} 43 \quad(5 \mathrm{I}-37-\mathrm{I} 2)$, veau $\mathrm{n}^{0} 44$ (54-37-9). Ces valeurs correspondent à celles trouvées par NDUMBE et al. (I964) dans le rumen de jeune Ruminant d'âge voisin. Selon ces auteurs, la forte proportion d'acide propionique et les faibles $\mathrm{pH}$ seraient dus à la présence d'acide lactique dans le rumen (nous n'avons pas dosé ce métabolite) ; la production réduite de salive chez le jeune Ruminant (KAx, I960) et les fortes concentrations des A. G. V. peuvent expliquer les faibles valeurs du $\mathrm{pH}$.

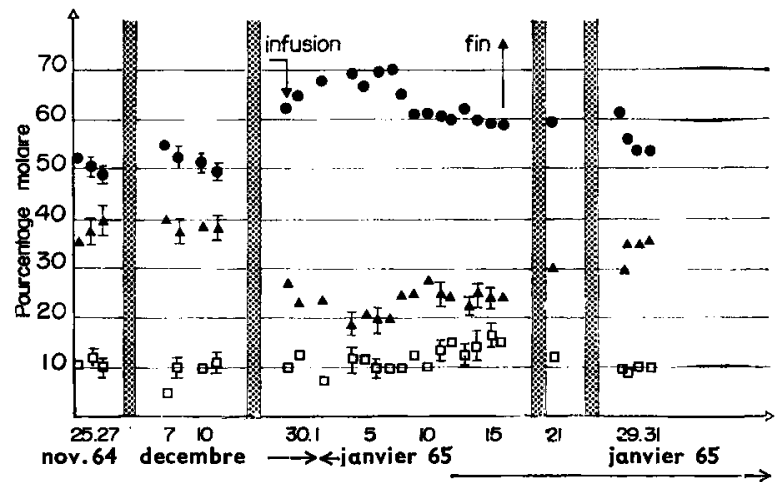

FIGURE 2. - Veau $n^{\circ} 43$. Évolution des pourcentages molaires d'acides gras volatils: acétique $(\bullet)$, propionique $(\Delta)$, acides supérieurs (ㅁ) en fonction du temps

Après l'infusion, pendant la période de contrôle $\left(2 \mathrm{~kg}\right.$ d'aliment $\mathrm{W}_{2}$ par jour) sur le veau no 43 , les pourcentages molaires des A. G. V. ont été voisins des précédents $(54,5-36,5-9)$; les taibles concentrations en A. G. V. $(88 \mathrm{mM} / 1)$ et le pH $(5,9)$ sont à mettre en rapport avec les quantités d'aliment et d'eau ( $18 \mathrm{~kg}$ ) consommées, l'augmentation probable de la production de salive et le développement allométrique du rumen.

L'infusion du mélange des A. G. V. a entraîné une augmentation nette du $\mathrm{pH}$ $(6,25$, moyenne pour les 2 veaux) et une diminution de la concentration en $A$. $G$. V. (r25 mM/1, moyenne pour le veau $\mathrm{n}^{\circ} 43$ ) ; de plus, le pourcentage molaire des A. G. V. est passé à $64,5-23,5-12$ (fig. 2). Une série de prélèvements effectués

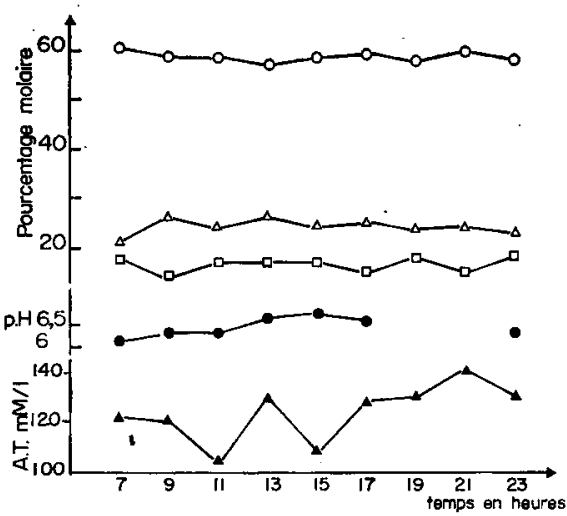

FIGURE 3. - Veau no 43. Évolution, au cours d'une journée (1 5-1-65), des $p H\left({ }^{\bullet}\right)$ acidité totale (A), pourcentages molaires des $A G V$ : acétique $(\mathrm{O})$, propionique $(\Delta)$, acides supérieurs $(\square)$ 
toutes les 2 heures, de 7 à 23 heures, en cours d'infusion, a montré que le $\mathrm{pH}$, la concentration en A. G. V. et le pourcentage de chaque acide variaient peu (fig. 3 ).

Pour mieux interpréter ces résultats, nous manquions de données (nombre d'animaux, dosages systématiques, ammoniaque, métabolites sanguins, volume du rumen). Nous pouvons néanmoins avancer quelques hypothèses pour expliquer les variations de pourcentages molaires des $A$. G. V. consécutives à l'infusion :

- la composition du mélange des A. G. V. dans le rumen est différente de celle des A. G. V. produits en raison de la différence des vitesses d'absorption (DANIELII et al., I945), (PFANDER et PhILLIPSON, I953),

- l'augmentation inexpliquée du $\mathrm{pH}$ et la diminution de la concentration en A. G. V. totaux auraient ralenti les vitesses d'absorption des A. G.JV. (GRAY, I948, SuTToN et al., I963) ; 1'acide butyrique et 1'acide propionique auraient été absorbés plus rapidement que 1'acide acétique,

- l'infusion de ce mélange des A. G. V. aurait entraîné des modifications des fermentations du rumen au profit de la production d'acide acétique, en relation avec l'augmentation du $\mathrm{pH}$ (LAMPILA, I964).

Rȩ̧u pour publication en mars 1967 .

\section{SUMMARY}

\section{EFFECTS OF A PERMANENT INFUSION OF A VOLATILE FATTY ACID MIXTURE IN THE RUMEN OF THE WEANED CALF}

Two rumen-canulated weaned calves about 4 months old and weighing 95 and $80 \mathrm{~kg}$ received a complete pelleted diet. A mixture of volatile fatty acids was permanently infused in the rumen for $I 7$ and II days respectively : the composition of this mixture was similar to that found in their rumen fluids : viz on a molar basis : acetic acid $5 \circ$, propionic acid 35 , butyric acid i 5 per cent.

The infusion of $200 \mathrm{~g}$ of this mixture solved in 3 l of water ( $\mathrm{pH}$ value : 2,0 ) induced a decrease in the amounts of food eaten ( 35 and 50 per cent). Rumen fluid $\mathrm{pH}$ increased from 5.2 to 6.25 ; total acidity decreased from $I 60$ to $I 25 \mathrm{mM} / \mathrm{l}$; the percents of volatile fatty acids altered from 54,5 , $36,9.0$ to $64.5,23.5$, I 2.0. Several explanations of the variations are discussed.

\section{RÉFÉRENCES BIBLIOGRAPHIQUES}

ARmstrong D. G., Blaxter K. L., 1965. Effects of acetic and propionic acids on energy retention and milk secretion in goats. Proc. of the 3 rd symposium held at Troon, may I964. In BLAXTER K. L. Energy metabolism, Academic Press, London, New York.

Bergman E. N,, Rein R. S., Murray M. G., Brockway J. M., Whitelaw F. G., 1965. Interconversions and production of volatile fatty acids in the sheep rumen. Biochem., J., 97, 53-58.

BlAXTER K. L., I962. The energy metabolism of ruminants. $185^{-261}$ Hutchinson Scientific an Technical I.ondon.

Danielli J. F., Hitchcock M. W. S., Marshall R. A., Phillipson A. T., 1945. The mechanism of absorption from the rumen as exemplified by the behaviour of acetic, propionic and butyric acids $J$. exper. Biol., 22-75.

Ndumbe R. D., Runcie K. V., McDonald P., ig64. The effect of early weaning on the blood sugar and rumen acid levels of the growing calf. Brit. J. Nutrit., 18, 29-38.

Elsden S. R., Hitchicook M. W. S., Marschall R. A., Phillipson A. T., i946. Volatile acids in the digesta of ruminants and others animals. J. exper. Biol., 22, 191.

Friedmann T, E., I938. The identification and quantitative determination of volatile alcohols and acids. J. biol. Chem., 128, 16r-1 84 . 
GaUsSERES B., 1965. Méthode de prélèvement automatique de la phase liquide des contenus de rumen. Ann. Biol. anim., Bioch., Biophys., 5, 407-4II.

Gray F. V., 1948. The absorption of volatile fatty acids from the rumen. II. The influence of $\mathrm{pH}$ on absorption. J. exper. Biol., 25, 135-144.

JAMES A. T., MARTIN A. J. P., 1952. Gas liquid partition chromatography : the separation and microestimation of volatile fatty acids from formic acid to dodecanoic acid. Biochem. J., 50, 679-69o.

KAY R. N. B., 1960. The development of parotid salivary secretion in young goats. J. Physiol., 150, 538-445.

LAMPILA M., 1964. Volatile fatty acids, $\mathrm{pH}$ and microbial activity in the rumen contents of the cow. Ann. Agric. Jenniae.

Pfander W. H., Phillipson A. T., 1953. The rates of absorption of acetic, propionic and $n$. butyric acids. $U$. Physiol., 122, 102-I 10.

SutTon J. D., McGilliaRd A. D., JACOBSON N. L., 1963. Fonctionnal development of rumen mucosa. I. Absorptive ability. J. Dairy Sci., 46, 426-436.

Tilley J. M. A., Canaway R. J., Terry R. A., I964. The estimation of volatile fatty acids by gas chromatography with automatic titration. Analyst, 89, 1058, 363-365. 